\title{
A Rapid In-Clinic Test Detects Acute Leptospirosis in Dogs with High Sensitivity and Specificity
}

\author{
Angeli Kodjo, ${ }^{1}$ Christophe Calleja, ${ }^{2}$ Michael Loenser, ${ }^{2}$ Dan Lin, ${ }^{3}$ and Joshua Lizer ${ }^{4}$ \\ ${ }^{1}$ Laboratoire des Leptospires, VetAgro Sup, Campus Veterinaire de Lyon, 1 avenue Bourgelat, 69280 Marcy l'Etoile, France \\ ${ }^{2}$ Zoetis Global Diagnostics, 100 Campus Drive, Florham Park, NJ 07932, USA \\ ${ }^{3}$ Zoetis Veterinary Medicine Research \& Development, Hoge Wei 10, 1930 Zaventem, Belgium \\ ${ }^{4}$ Zoetis Veterinary Medicine Research \& Development, 333 Portage Street, Kalamazoo, MI 49007, USA
}

Correspondence should be addressed to Joshua Lizer; joshua.lizer@zoetis.com

Received 9 November 2015; Accepted 9 March 2016

Academic Editor: Andre Talvani

Copyright (C) 2016 Angeli Kodjo et al. This is an open access article distributed under the Creative Commons Attribution License, which permits unrestricted use, distribution, and reproduction in any medium, provided the original work is properly cited.

\begin{abstract}
A rapid IgM-detection immunochromatographic test (WITNESS ${ }^{\circledR}$ Lepto, Zoetis) has recently become available to identify acute canine leptospirosis at the point of care. Diagnostic sensitivity and specificity of the test were evaluated by comparison with the microscopic agglutination assay (MAT), using a positive cut-off titer of $\geq 800$. Banked serum samples from dogs exhibiting clinical signs and suspected leptospirosis were selected to form three groups based on MAT titer: (1) positive $(n=50)$; (2) borderline $(n=35)$; and (3) negative $(n=50)$. Using an analysis to weight group sizes to reflect French prevalence, the sensitivity and specificity were $98 \%$ and $93.5 \%$ (88.2\% unweighted), respectively. This test rapidly identifies cases of acute canine leptospirosis with high levels of sensitivity and specificity with no interference from previous vaccination.
\end{abstract}

\section{Introduction}

Leptospirosis is a global bacterial zoonotic disease affecting humans and wild and domestic animals, including dogs. Clinical signs of leptospirosis in dogs are nonspecific, typically including renal and/or hepatic dysfunction, and can manifest as subclinical, chronic, or acute infections, sometimes with fatal outcomes. Infected animals excrete Leptospira in their urine and can pose a risk to humans who become infected through damaged skin or via the conjunctiva or mucosa, causing a potentially fatal disease [1].

The reference serological test for diagnosis is the microscopic agglutination test (MAT). Because the test is complex to perform and interpret, serum must be sent to a reference laboratory [1]. As such, MAT tends to be the last resort in first-opinion practice. An immunochromatographic test (WITNESS Lepto, Zoetis) has been developed, offering the advantage of a result in $10 \mathrm{~min}$. The test uses whole cell antigen extracts of L. kirschneri serovar Grippotyphosa and L. interrogans serovar Bratislava to detect canine IgM made in response to infection. These serovars were selected as they are of clinical relevance and relative prevalence worldwide [2-8]. However, unpublished data from internal studies and the current study suggest broad serovar reactivity on the test, likely due to conserved antigens across pathogenic Leptospira. The objective of the present study was to evaluate the sensitivity (Se) and specificity (Sp) of this new test in client-owned French dogs as compared to MAT.

\section{Materials and Methods}

All samples in this study were taken from an established bank of clinical samples submitted by French veterinarians to the Laboratoire des Leptospires (VetAgro Sup, Marcy L'Etoile, France) for diagnostic testing because leptospirosis was suspected. A total of 135 canine serum samples were tested using WITNESS Lepto retrospectively compared to MAT. Based on previous MAT results, the samples were divided into three groups: (1) a positive group $(n=50)$ defined as having a MAT titer $\geq 800$ for any of the 19 serovars used in the test and a diagnosis of leptospirosis; (2) a borderline group $(n=35)$ defined as having a MAT titer $<800$ for at least 
TABLE 1: Weighted prevalence rates based on French data ${ }^{a}$.

\begin{tabular}{lccc}
\hline Group & $n$ in present study & French prevalence $^{\text {a }}$ & Weighted $n$ in present study \\
\hline Positive (MAT titer $\geq 800)$ & 50 & $25 \%$ & 33.75 \\
Borderline (MAT titer $<800)$ & 35 & $17 \%$ & 22.95 \\
Negative (MAT titer negative or vaccinal $\leq 400)$ & 50 & $58 \%$ & 78.3 \\
\hline
\end{tabular}

MAT: microscopic agglutination test.

${ }^{a}$ Renaud et al., 2013 [7].

TABLE 2: MAT versus WITNESS Lepto: $2 \times 2$ table of weighted and unweighted results used for the calculation of sensitivity and specificity.

(a) Unweighted results

\begin{tabular}{lcc}
\hline WITNESS Lepto & MAT positive $(n)$ & MAT negative $(n)$ \\
\hline Positive & TP: 49 (positive group) & FP: 10 (borderline group) \\
Negative & FN: 1 (positive group) & TN: 25 (borderline group), \\
& & 50 (negative group) \\
\hline
\end{tabular}

(b) Weighted data used to calculate Se and Sp

\begin{tabular}{lcr}
\hline WITNESS Lepto & MAT positive $(n)$ & MAT negative $(n)$ \\
\hline Positive & TP: 33.075 & FP: 6.55 \\
Negative & FN: 0.675 & TN: 94.45 \\
& Sensitivity: $98 \%$ & Specificity: $93.5 \%$ \\
\hline
\end{tabular}

MAT: microscopic agglutination test; Se: sensitivity; Sp: specificity; TP: true positive; TN: true negative; FP: false positive; FN: false negative.

one nonvaccine serovar and an inconclusive diagnosis; and (3) a negative group $(n=50)$ defined as having MAT titers completely negative or titers $\leq 400$ only for vaccine serovars and a negative diagnosis of leptospirosis. The samples were tested in a random order and the technician performing the test was blinded. Each sample was tested using a prototype of WITNESS Lepto, following the manufacturer's instructions. To perform the test, $5 \mu \mathrm{L}$ of sample was added to the sample well of the test cassette. Four drops $(\sim 140 \mu \mathrm{L})$ of a chase buffer was then added and the test was allowed to develop for 10 minutes, at which time the result was interpreted. The formation of only a red control line indicates a negative test, while the formation of a red test line and control line indicates a positive test.

Because the samples in each group were selected to ensure a sufficient sample size, the distribution of the samples (positive, borderline, and negative) in this study did not reflect the empirically determined prevalence in France [7]. To adjust for this in the calculation of Se and Sp, the group sample sizes were weighted to reflect the prevalence rate reported by Renaud et al., 2013 (Table 1). The weighting was achieved by multiplying the French prevalence for each group by the total $n(n=135)$. The percentage of true and false positives or negatives per group was calculated using the original study data. The percentages were then applied to the weighted groups for the purposes of Se and Sp calculations.

Diagnostic Se and Sp of WITNESS Lepto were evaluated by comparison with MAT, using a positive cut-off titer of $\geq 800$. All the samples in the positive group were defined as positive, whereas the samples in the borderline and negative groups were defined as negative. The analysis determined Se and Sp with 95\% Jeffreys' confidence intervals (CI) $[9,10]$.

\section{Results and Discussion}

Forty-nine of $50(49 / 50)$ samples in the MAT positive group were positive, while 25/35 MAT borderline samples and all 50 MAT negative samples were negative on WITNESS Lepto (Table 2(a)). Using the weighted distribution, Se was $98 \%$ (95\% CI 88.7 to $99.9 \%$ ) and Sp was $93.5 \%$ (95\% CI 87.4 to $97.1 \%)$. With no weighting to the sample distribution, the Se remained at $98 \%$ and the $\mathrm{Sp}$ was $88.2 \%$. The data is summarized in Table 2(b). Forty-two of the 50 MAT negative dogs had previously received a Leptospira vaccine with 38 of them being within a year of vaccination, suggesting that previous vaccination did not interfere with a correct test result.

The level of agreement between WITNESS Lepto and MAT was very high for samples that were either MAT positive or MAT negative. All but one of the samples where the WITNESS result differed with MAT came from the borderline group. The borderline samples were considered negative as their MAT titers were not $\geq 800$. With this classification, some samples in the borderline group were still likely to be truly infected. An IgM immunoblot assay had $88 \%$ sensitivity in the first three days of human leptospirosis, compared to $2 \%$ sensitivity for the MAT [11]. Another human IgM ELISA detected infection in $29 \%$ of the cases before MAT had detectable titers [12]. Thus, WITNESS Lepto may have been correctly identifying positive samples in the borderline group before they reached a MAT titer of $\geq 800$. This emphasizes the difficulty in the interpretation of a single MAT in practice. The sensitivity and specificity of MAT increase considerably when samples collected from the animal one or two weeks apart can be tested [13]. However, client compliance can 
be difficult due to an extra trip to the clinic at extra cost. The WITNESS test provides a time-saving, easy to interpret, and economical solution in this regard. The test provides additional advantages to the MAT because the test is safe and does not require working with live organism, as is the case with the MAT. The convenience of testing in the clinic provides immediate identification of infected dogs, allowing for proper quarantine and handling protocols to be implemented which reduces the risk of transmission to humans and other animals. Whereas the MAT detects both IgM and IgG, WITNESS Lepto detects only IgM, simplifying result interpretation and negating cross-reactivity with IgG antibody from dogs with a previous Leptospira vaccination [14].

\section{Conclusions}

In conclusion, this study indicates that WITNESS Lepto is a reliable test with high levels of sensitivity and specificity, as compared to the MAT, for the diagnosis of acute leptospirosis in dogs that are showing compatible clinical signs. With its ease of use and immediate result, it can transform the veterinarian's case management by enabling earlier diagnosis and treatment or ruling out of leptospirosis, all to the benefit of both the patient and the public health.

\section{Competing Interests}

The authors Christophe Calleja, Michael Loenser, Dan Lin, and Joshua Lizer are employed by Zoetis and were responsible for the experimental design and analysis of the data. Angeli Kodjo conducted the testing and selected the individual samples to be used under a research agreement fully funded by Zoetis.

\section{References}

[1] P. N. Levett, "Leptospirosis: a forgotten zoonosis?" Clinical and Applied Immunology Reviews, vol. 4, no. 6, pp. 435-448, 2004.

[2] R. D. Blazius, P. R. T. Romão, E. M. C. G. Blazius, and O. S. da Silva, "Occurrence of Leptospira spp. soropositive stray dogs in Itapema, Santa Catarina, Brazil," Cadernos de Saúde pública, vol. 21, no. 6, pp. 1952-1956, 2005.

[3] P. Boutilier, A. Carr, and R. L. Schulman, "Leptospirosis in dogs: a serologic survey and case series 1996 to 2001," Veterinary Therapeutics, vol. 4, no. 2, pp. 178-187, 2003.

[4] R. Gautam, C.-C. Wu, L. E. Guptill, A. Potter, and G. E. Moore, "Detection of antibodies against Leptospira serovars via microscopic agglutination tests in dogs in the United States, 2000-2007," Journal of the American Veterinary Medical Association, vol. 237, no. 3, pp. 293-298, 2010.

[5] V. Geisen, C. Stengel, S. Brem, W. Müller, C. Greene, and K. Hartmann, "Canine leptospirosis infections-clinical signs and outcome with different suspected Leptospira serogroups (42 cases)," Journal of Small Animal Practice, vol. 48, no. 6, pp. 324328, 2007.

[6] M. Oliveira Lavinsky, R. A. Said, G. M. R. Strenzel, and H. Langoni, "Seroprevalence of anti-Leptospira spp. antibodies in dogs in Bahia, Brazil," Preventive Veterinary Medicine, vol. 106, no. 1, pp. 79-84, 2012.
[7] C. Renaud, S. Andrews, Z. Djelouadji et al., "Prevalence of the Leptospira serovars bratislava, grippotyphosa, mozdok and pomona in French dogs," Veterinary Journal, vol. 196, no. 1, pp. 126-127, 2013.

[8] J. E. Stokes, J. B. Kaneene, W. D. Schall et al., "Prevalence of serum antibodies against six Leptospira serovars in healthy dogs," Journal of the American Veterinary Medical Association, vol. 230, no. 11, pp. 1657-1664, 2007.

[9] H. Jeffreys, Theory of Probability, Oxford University Press, 1939.

[10] H. Jeffreys, "An invariant form for the prior probability in estimation problems," Proceedings of the Royal Society of London, Series A: Mathematical and Physical Sciences, vol. 186, no. 1007, pp. 453-461, 1946.

[11] J. E. Sykes, K. Hartmann, K. F. Lunn, G. E. Moore, R. A. Stoddard, and R. E. Goldstein, "2010 ACVIM small animal consensus statement on leptospirosis: diagnosis, epidemiology, treatment, and prevention," Journal of Veterinary Internal Medicine, vol. 25, no. 1, pp. 1-13, 2011.

[12] W. E. Winslow, D. J. Merry, M. L. Pirc, and P. L. Devine, "Evaluation of a commercial enzyme-linked immunosorbent assay for detection of immunoglobulin $\mathrm{M}$ antibody in diagnosis of human leptospiral infection," Journal of Clinical Microbiology, vol. 35, no. 8, pp. 1938-1942, 1997.

[13] S. Schuller, T. Francey, K. Hartmann et al., "European consensus statement on leptospirosis in dogs and cats," Journal of Small Animal Practice, vol. 56, no. 3, pp. 159-179, 2015.

[14] P. N. Levett, "Leptospirosis," Clinical Microbiology Reviews, vol. 14, no. 2, pp. 296-326, 2001. 


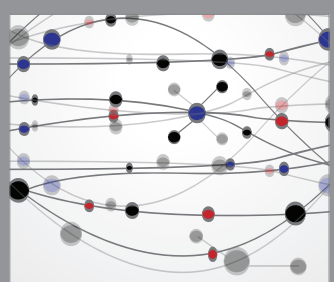

The Scientific World Journal
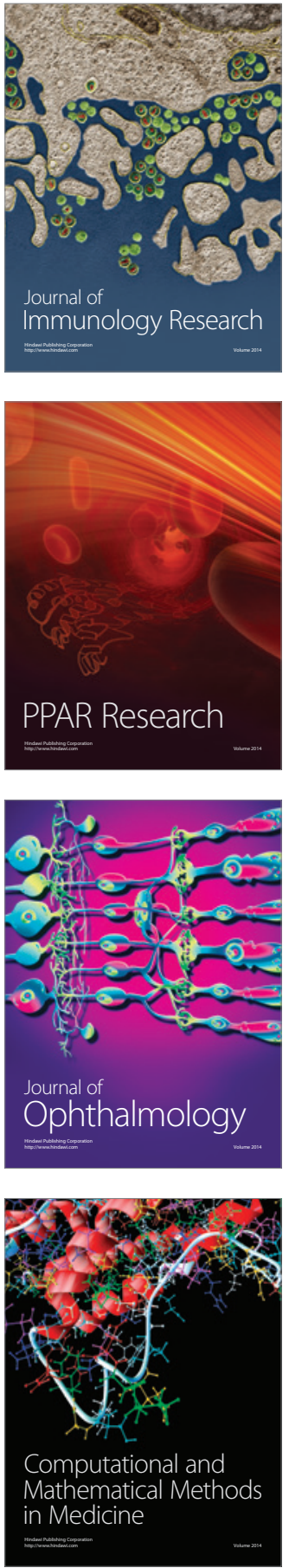

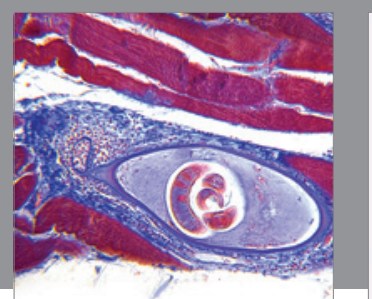

Gastroenterology Research and Practice

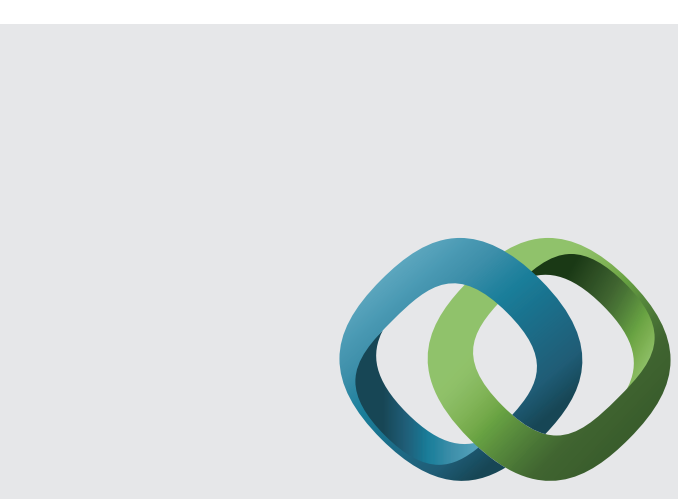

\section{Hindawi}

Submit your manuscripts at

http://www.hindawi.com
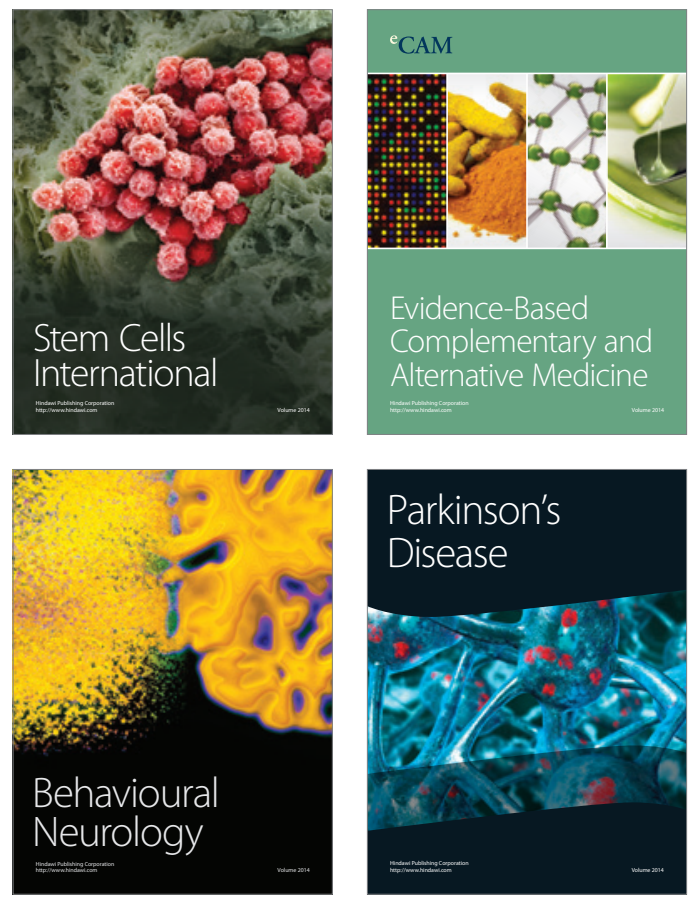
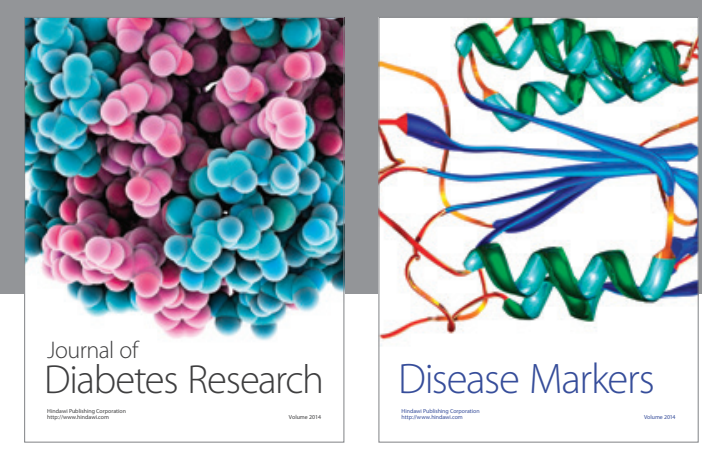

Disease Markers
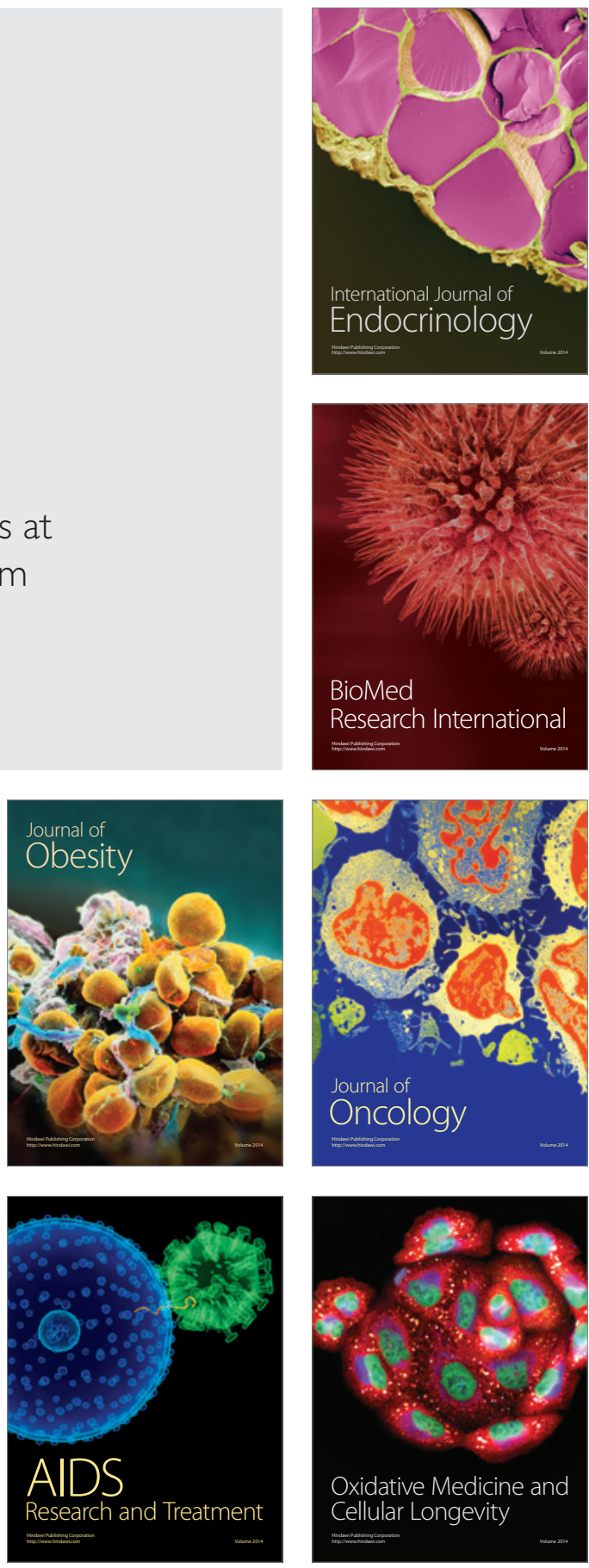